\title{
Use of caudal pancreatectomy as a novel adjunct procedure to proximal splenorenal shunt in patients with noncirrhotic portal hypertension: A retrospective cohort study
}

\author{
Shahana Gupta, Biju Pottakkat, Raja Kalayarasan, Gnanasekaran Senthil, Pagadala Naga Balaji Nitesh \\ Department of Surgical Gastroenterology, Jawaharlal Institute of Postgraduate Medical Education and Research, Puducherry, India
}

Backgrounds/Aims: Proximal splenorenal shunt (PSRS) is considered a one-time treatment for noncirrhotic portal hypertension $(\mathrm{NCPH})$ to prevent recurrent upper gastrointestinal (UGI) hemorrhage and long-term complications. Long-term shunt patency is necessary to achieve these. The lie of the shunt is a contributing factor to early shunt thrombosis. We investigated the role of resection of the distal tail of pancreas (caudal pancreatectomy [CP]) in improving the lie of shunt and decreasing shunt thrombosis.

Methods: This was a retrospective cohort study of patients with NCPH who underwent PSRS between 2014-2020 in JIPMER, Puducherry, India. CP was performed in patients with a long tail of pancreas, with the tip of pancreatic tail extending up to splenic hilum on preoperative CT. Perioperative parameters and shunt patency rate of patients who underwent PSRS with CP (Group A) were compared with patients undergoing conventional PSRS (Group B). Statistical analysis was performed using the Mann-Whitney U test and $\chi^{2}$ test.

Results: Eighty four patients with NCPH underwent PSRS (extrahepatic portal vein obstruction = 39; noncirrhotic portal fibrosis = $45)$. Blood loss was lower $(p=0.002)$ and post-shunt fall in portal pressure higher $(p=0.002)$ in Group A. Shunt thrombosis rate was lower $(p=0.04)$ while rate of complete variceal regression $(p=0.03)$ and biochemical pancreatic leak $(p=0.01)$ were higher in Group A.There was no clinically relevant pancreatic fistula in either group.

Conclusions: $\mathrm{CP}$ is a safe and useful technique for reducing shunt thrombosis after PSRS in patients with NCPH by improving the lie of shunt.

Key Words: Caudal pancreatectomy; Noncirrhotic portal hypertension; Proximal splenorenal shunt; Lie of the shunt; Shunt thrombosis

\section{INTRODUCTION}

Received: July 12, 2021, Revised: November 4, 2021, Accepted: November 8, 2021

Corresponding author: Biju Pottakkat Department of Surgical Gastroenterology, Jawaharlal Institute of Postgraduate Medical Education and Research, Jipmer Campus Rd, Dhanvantari Nagar, Puducherry 605006, India

Tel: +91-7598566982, Fax: +91-8179705152, E-mail: bijupottakkat@gmail.com ORCID: https://orcid.org/0000-0002-8474-0270

Copyright (C) The Korean Association of Hepato-Biliary-Pancreatic Surgery This is an Open Access article distributed under the terms of the Creative Commons Attribution Non-Commercial License (http://creativecommons.org/licenses/by-nc/4.0) which permits unrestricted non-commercial use, distribution, and reproduction in any medium, provided the original work is properly cited.
Proximal splenorenal shunt (PSRS) is a commonly performed operative procedure for noncirrhotic portal hypertension (NCPH). It is considered a one-time treatment option to prevent upper gastrointestinal (UGI) hemorrhage from gastro-esophageal and ectopic varices. Shunt surgery also prevents long-term complications of portal hypertension such as portal biliopathy $[1,2]$. Long-term patency of the shunt is necessary to achieve the aforesaid objectives. Numerous factors have been reported to contribute to shunt thrombosis [3]. Amongst these, a proper lie of the splenic vein (SV) with respect to the left renal vein is a factor in the technical success of PSRS. Ours is a high-volume center for surgical treatment of portal hypertension $[4,5]$. We have observed that a long tail of pancreas im- 
pinged on the splenorenal anastomosis can result in kinking of the PSRS, thus altering its lie. Hence, we selectively performed resection of the distal-most portion of the tail of pancreas (caudal pancreatectomy [CP]) to increase the available length of the SV and improve the lie of the shunt.

This study focused on the role of CP in improving the lie of the shunt and in reducing the incidence of shunt thrombosis in patients with NCPH undergoing PSRS.

\section{PATIENTS AND METHODS}

This was a retrospective cohort study of patients with NCPH who underwent PSRS between 2014-2020 in Jawaharlal Institute of Postgraduate Medical Education and Research (JIPMER), Puducherry, a tertiary care hospital and an Institute of National Importance in India. This study was approved by the Institute PG Research Monitoring and Ethics Committee (Ref No: JIP/IEC/2014/10/478 Registration Number of IEC: ECR/342/INST/PY/2013). All participants gave informed consent before inclusion in this study. Groups A and B comprised of patients who underwent CP with PSRS and conventional PSRS alone, respectively. Demographic, clinical, and endoscopic parameters of both groups were compared (Table 1).

\section{Investigations and preoperative preparation}

Indications for shunt surgery in patients with $\mathrm{NCPH}$ have been previously reported $[3,4]$. Patients requiring shunt surgery were evaluated by UGI endoscopy, Doppler study of the porto-splenomesenteric system, Acoustic resonance forced impulse scan, and computed tomography (CT) portovenogram. The presence of a long tail of pancreas especially with the tip of the pancreatic tail extending up to the splenic hilum on pre-operative CT portovenogram (Fig. 1) was used as a criterion for selecting patients for CP. The coagulation workup for procoagulant disorders was not done for any patient due to logistic reasons. All patients received pneumococcal, meningo-

Table 1. Demographic, clinical, and endoscopic parameters

\begin{tabular}{lccc}
\hline \multicolumn{1}{c}{ Characteristic } & Group A & Group B & $p$-value \\
\hline Age (yr) & $19(15-45)$ & $22(12-55)$ & $0.70^{\text {a) }}$ \\
Sex (male : female) & $7: 10$ & $23: 44$ & $0.80^{\text {b) }}$ \\
Diagnosis (EHPVO/NCPF) (ratio) & 0.9 & 0.9 & $0.82^{\text {b) }}$ \\
Symptoms (UGI bleed) & 13 & 62 & $0.14^{\text {b) }}$ \\
Symptom duration (mon) & $98(5-190)$ & $108(1-240)$ & $0.54^{\text {a) }}$ \\
Grade of esophageal varices & $8: 9$ & $37: 30$ & $0.74^{\text {b) }}$ \\
$\quad$ at presentation ( $\leq$ II : $>$ II) & & & \\
\hline
\end{tabular}

Values are presented as median (range) or number only.

Group A, patients who underwent proximal splenorenal shunt (PSRS) with caudal pancreatectomy $(n=17)$; Group B, patients who underwent conventional PSRS ( $n=67)$; EHPVO, extrahepatic portal vein obstruction; NCPF, noncirrhotic portal fibrosis; UGI, upper gastrointestinal.

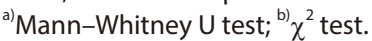

coccal, and Haemophilus influenzae vaccines preoperatively.

\section{Operative procedure}

The technique of PSRS has been previously reported [2-4,6]. PSRS was performed through the left trap door incision with the patient in a supine position with slight elevation of the left flank. Splenic artery was identified at the upper border of the pancreas and ligated in continuity. Spleen was removed only after dissecting the SV completely and preserving adequate length of SV.

$\mathrm{CP}$ was performed in patients with a long tail of the pancreas, especially when the tip of the pancreatic tail extended up to the splenic hilum as seen in preoperative CT portovenogram (Fig. 1), and further assessed intraoperatively by the operating surgeon taking into consideration the effectiveness of $\mathrm{CP}$ in increasing the available length of the SV and eventually improving the lie of the shunt. In these patients, after performing splenectomy, approximately $2-3 \mathrm{~cm}$ of the pancreatic tail was resected using a laparoscopic linear cutter (Fig. 2) to increase the available length of the SV (Fig. 3). The stump of the pancreas was reinforced with 4-0 polypropylene sutures to secure hemostasis and to prevent pancreatic duct leak. Gerota's fascia was dissected and the left kidney was exposed at the medial aspect. The left renal vein was identified and dissected out. The left gonadal vein was ligated and divided close to the left renal vein. The end of the SV was anastomosed with the side of the left renal vein in a continuous fashion using 5-0 or 6-0 Prolene. The lie of the shunt is related to the proper alignment of the $\mathrm{SV}$, which is a mobile structure with respect to the left renal vein, a fixed structure. The most acceptable position is to align the midpoint of the anterior wall of the SV to the left corner of the cut edge of the left renal vein. This was very well achieved by removing the fixity of the SV to the tail of the pancreas. CP thus improved the lie of the shunt by removing this fixity of SV to the pancreatic tail and attaining the appropriate align-

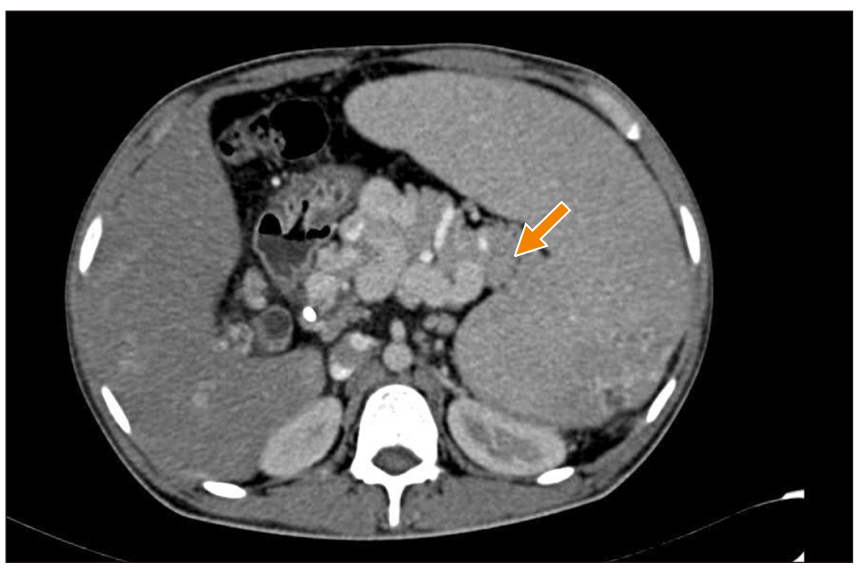

Fig. 1. Computed tomography portovenogram showing the long tail of pancreas with tip of pancreatic tail extending up to splenic hilum (arrow). 


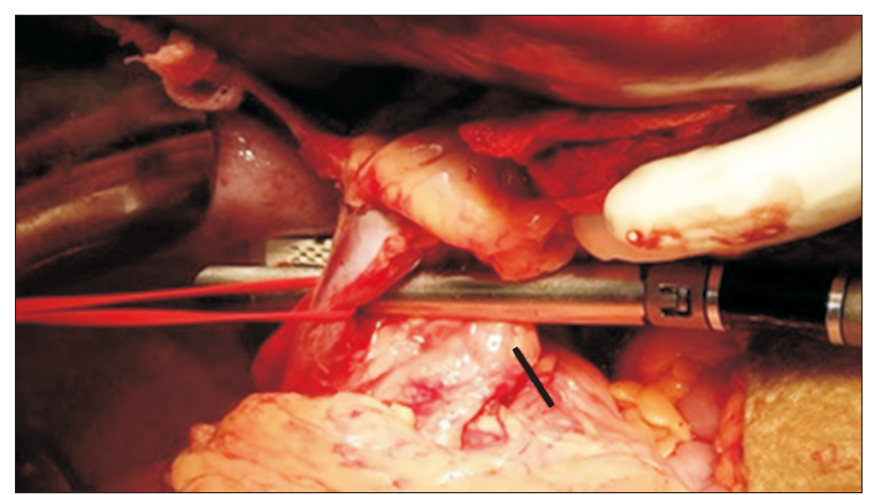

Fig. 2. Caudal pancreatectomy being performed using a laparoscopic linear cutter. The black line indicates the distal end of the pancreas. The splenic vein is looped (red loop).

ment as mentioned above. Once this acceptable position was attained, the lie was considered to be satisfactory (Fig. 4).

Intraoperatively, pre- and post-shunt portal pressures were measured using a cannula attached to an omental vein and connected to a pressure transducer. The SV and shunt diameter were measured using a sterile scale.

Drain fluid amylase was assessed routinely for all patients on postoperative day-3. Postoperative pancreatic fistula was defined and graded using the criteria recommended by the International Study Group of Pancreatic Surgery [7].

Histopathological examination of the SV wall was performed for all patients to assess pathological abnormalities in the SV [5].

\section{Postoperative follow-up}

Postoperatively, all patients received oral anticoagulants for two years. They were followed up at 1, 3, 6, 9, 12 months, and every six months thereafter for five years with prothrombin time, platelet count, UGI endoscopy, Doppler study of the por-

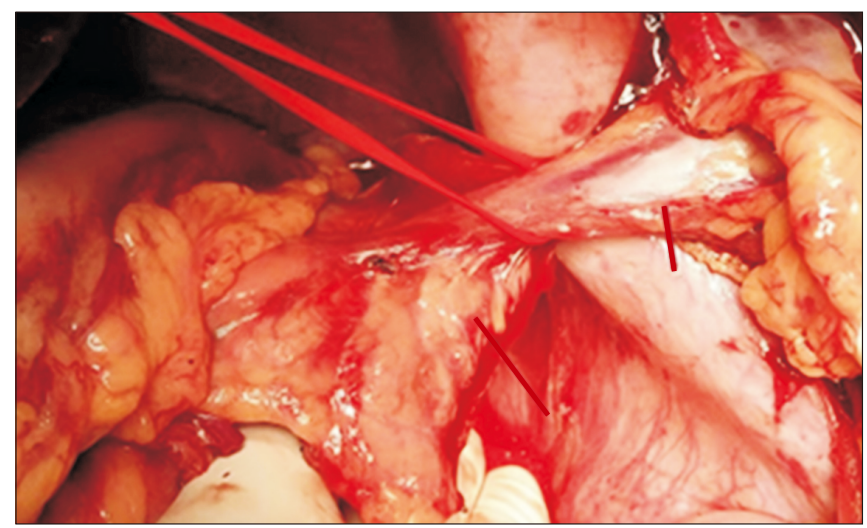

Fig. 3. After caudal pancreatectomy. Long line indicates cut end of the pancreas. Short line indicates the adequate length of splenic vein.

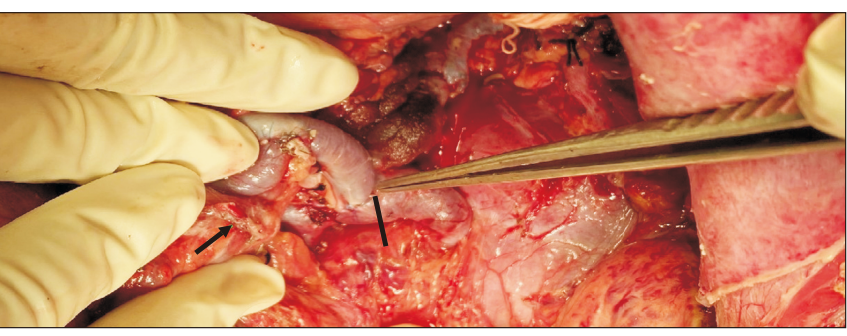

Fig. 4. After caudal pancreatectomy and proximal splenorenal shunt, the satisfactory lie of the shunt. Black line shows the site of splenorenal anastomosis; arrow indicates the cut end of pancreas.

to-splenomesenteric system, and shunt. CT portovenogram was performed for patients in whom shunt thrombosis was suspected on Doppler evaluation. Shunt thrombosis rates at 3 years of follow-up were considered for comparison.

\section{Statistical analysis}

Categorical data such as the status of complete variceal regression, pancreatic leak, rate of shunt thrombosis, and lie of shunt are expressed as frequency and proportion. Quantitative data such as SV diameter, shunt diameter, post shunt fall in portal pressure, operative time, and intraoperative blood loss are expressed as median and range. For statistical analysis, Mann-Whitney $U$ test was used for quantitative data and $\chi^{2}$ test was used for categorical data. All statistical analyses were performed using the statistical program GraphPad INSTAT version 3 (GraphPad Software, Inc., La Jolla, CA, USA). $p<0.05$ was considered significant.

\section{RESULTS}

\section{Study group}

This study involved 86 patients with NCPH. Of these, 40 patients had extrahepatic portal vein obstruction (EHPVO) and 46 had noncirrhotic portal fibrosis (NCPF). PSRS was performed in 84/86 patients. The remaining two (EHPVO : $\mathrm{NCPF}=1: 1$ ) were found to have unshuntable SVs during surgery. PSRS was abandoned for them. These two patients were therefore excluded from data analysis. Thus, the analysis was based on 84 patients (EHPVO $: \mathrm{NCPF}=39: 45$ ). Seventeen patients (Group A) underwent CP with PSRS (EHPVO : NCPF = $8: 9$ ) and the rest 67 (Group B) underwent conventional PSRS (EHPVO : NCPF $=31: 36$ ).

The majority of patients were females $(\mathrm{n}=54,64.2 \%)$. Median (range) age was 22 years (range, $12-55$ years). Symptom duration was 108 months (range, 1-240 months). The most common presentation in both EHPVO and NCPF patients was UGI hemorrhage (89.3\%). Clinical and demographic characteristics of the two groups did not differ significantly (Table 1). Hypersplenism was present in all 84 patients. 
Table 2. Intraoperative parameters in Group A and Group B

\begin{tabular}{llcc}
\hline \multicolumn{1}{c}{ Parameter } & Group A & Group B & $p$-value \\
\hline SV diameter (mm) & $12(8-16)$ & $11(8-15)$ & $0.21^{\text {a) }}$ \\
Shunt diameter (mm) & $10(8-13)$ & $10(8-13)$ & $0.49^{\text {a) }}$ \\
$\begin{array}{c}\text { Post shunt fall in portal } \\
\text { pressure (mmHg) }\end{array}$ & $16(13-22)$ & $14(9-19)$ & $0.002^{\text {a) }}$ \\
$\begin{array}{l}\text { Operative time (min) } \\
\text { Intraoperative } \\
\text { blood loss (mL) }\end{array}$ & $275(200-360)$ & $260(180-360)$ & $0.38^{\text {a) }}$ \\
Satisfactory lie of shunt & $15(88.2)$ & $42(62.6)$ & $0.002^{\text {a) }}$ \\
\hline
\end{tabular}

Values are presented as median (range) or number (\%).

Group A, patients who underwent proximal splenorenal shunt (PSRS) with caudal pancreatectomy $(n=17)$; Group $B$, patients who underwent conventional PSRS ( $n=67)$; SV, splenic vein.

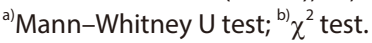

\section{Intraoperative and postoperative parameters}

Of intraoperative parameters (Table 2), SV diameter, shunt diameter, and operative time did not differ significantly between the two groups. Difference was observed in intraoperative blood loss $(p=0.002)$ and post-shunt fall in portal pressure $(p=0.002)$. Blood loss in $14.9 \%(10 / 67)$ patients of Group B was in the range of $900-1,600 \mathrm{~mL}$, which was at the upper end of the distribution of blood loss values in this group (200-1,600 $\mathrm{mL}$ ), well outside the corresponding range in Group A (200-500 $\mathrm{mL})$.

A satisfactory lie was achieved in $67.9 \%(57 / 84)$ patients. In Group A, the lie of the shunt was satisfactory in $88.2 \%(15 / 17)$ patients (Fig. 4, 5). The remaining 11.8\% (2/17) patients did not have a satisfactory lie even after CP. They developed shunt thrombosis during follow-up. In Group B, a satisfactory lie as defined in Section 2.2 could not be achieved in 25 patients

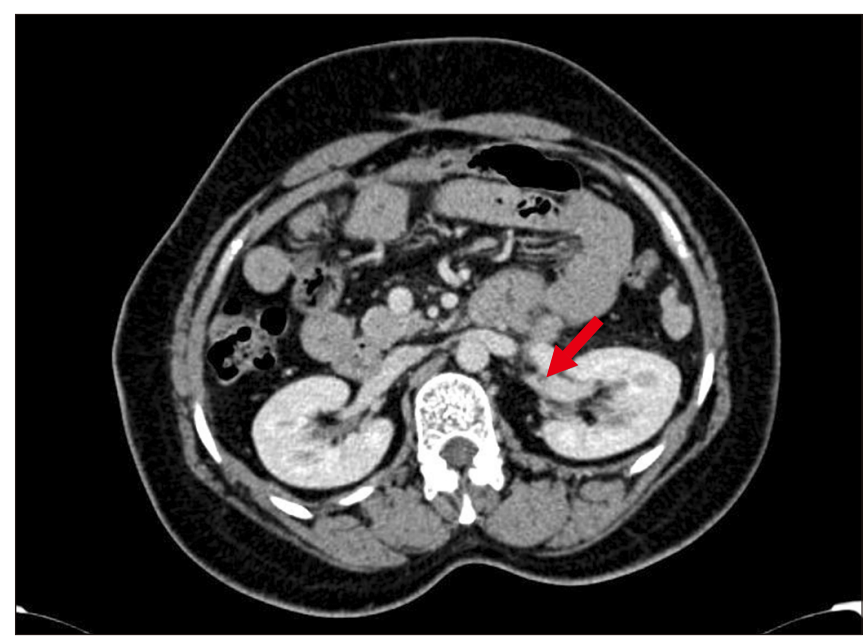

Fig. 5. Postoperative computed tomography portovenogram showing satisfactory lie of shunt after caudal pancreatectomy. Red arrow indicates the site of splenorenal anastomosis.
Table 3. Postoperative parameters in Group A and Group B

\begin{tabular}{lccc}
\hline \multicolumn{1}{c}{ Variable } & Group A & Group B & $p$-value \\
\hline Complete variceal regression & $15(88.2)$ & $40(59.7)$ & 0.03 \\
Biochemical pancreatic leak & $4(23.5)$ & $3(4.5)$ & 0.01 \\
Shunt thrombosis & $2(11.8)$ & $25(37.3)$ & 0.04 \\
\hline
\end{tabular}

Values are presented as number (\%).

Group A, patients who underwent proximal splenorenal shunt (PSRS) with caudal pancreatectomy $(n=17)$; Group B, patients who underwent conventional PSRS $(\mathrm{n}=67)$.

${ }^{\text {a) }} \chi^{2}$ test.

who were operated upon before our team started performing CP. They developed shunt thrombosis during follow-up. The lie of the remaining 42 patients in Group B who had a patent shunt was found to be satisfactory. A satisfactory lie was hence achieved in a higher proportion of patients in Group A $(88.2 \%$ vs. $62.6 \% ; p=0.04$ ) (Table 2).

Biochemical pancreatic leak rate was higher $(p=0.01)$ in Group A $(23.5 \%$; 4/17) (Table 3). No patients in the study group had a clinically relevant postoperative pancreatic fistula. The majority $(80 / 84,95.2 \%)$ of patients had transient ascites postoperatively. Two patients in Group A and two patients in Group $B$ developed wound hematoma which was managed conservatively (Clavien-Dindo Grade 1) [8]. Median hospital stay was 7 days (range, 6-14 days) in both groups.

The significance of possible confounding factors affecting shunt thrombosis is given in Table 4. SV of all patients in the study group was found to be pathological [5]. The technique of anastomosis and type of suture material was also the same in all patients. Therefore, these were not considered as confounding factors.

Table 4. Confounding factors affecting shunt thrombosis

\begin{tabular}{|c|c|c|c|}
\hline Factor & $\begin{array}{l}\text { Thrombosed } \\
\text { shunt } \\
(n=27)\end{array}$ & $\begin{array}{l}\text { Patent } \\
\text { shunt } \\
(n=57)\end{array}$ & $p$-value \\
\hline Diagnosis (EHPVO : NCPF) & $13: 14$ & $26: 31$ & $0.83^{\mathrm{a})}$ \\
\hline $\begin{array}{l}\text { Grade of } \\
\text { esophageal varices at } \\
\text { presentation }(\leq \mathrm{II}:>\mathrm{II})\end{array}$ & $13: 14$ & $25: 32$ & $0.89^{\mathrm{a})}$ \\
\hline SV diameter (mm) & $12(8-16)$ & $11(8-16)$ & $0.21^{\mathrm{b})}$ \\
\hline Shunt diameter $(\mathrm{mm})$ & $10(8-13)$ & $10(8-13)$ & $0.49^{b)}$ \\
\hline $\begin{array}{l}\text { Interval between shunt } \\
\text { surgery \& evaluation for } \\
\text { thrombosis (yr) }\end{array}$ & $1(0.9-3.0)$ & $1.2(0.7-3.0)$ & $0.38^{\mathrm{b})}$ \\
\hline $\begin{array}{l}\text { Post splenectomy } \\
\text { platelet count } \\
\left(\text { lakhs } / \mathrm{mm}^{3}\right)\end{array}$ & $3.08(1.0-9.0)$ & $2.85(1.0-7.0)$ & $0.75^{\mathrm{b})}$ \\
\hline
\end{tabular}

Values are presented as number only or median (range).

EHPVO, extrahepatic portal vein obstruction; NCPF, noncirrhotic portal fibrosis; SV, splenic vein.

a) $\chi^{2}$ test; ${ }^{b}$ Mann-Whitney $U$ test. 


\section{Follow-up}

All patients were followed up for a minimum of three years. The overall shunt thrombosis rate at about three years follow-up was $32.1 \%$ (27/84). Shunt thrombosis rate was lower in Group A (11.8\% vs. 37.3\%, $p=0.04)$. In Group A, 11.8\% (2/17) patients developed shunt thrombosis at 8 and 12 months respectively, and presented with recurrent UGI hemorrhage. In Group B, 37.3\% (25/67) patients had shunt thrombosis (Table 3).

Overall, 65.5\% (55/84) patients had complete regression of varices on UGI endoscopy while $34.5 \%$ (29/84) had a reduction in grade of varices. Complete variceal regression was higher in Group A $(p=0.03)$ (Table 3$)$. Of the 29 patients with a reduction in grade of varices, 27 (Group A: Group B = 2: 25) had shunt thrombosis while the remaining two patients (Group B) had patent shunts. All 84 had a correction of pancytopenia. None of the patients developed any features of endocrine or exocrine insufficiency during follow-up.

\section{DISCUSSION}

Shunt thrombosis is a common and important complication of PSRS reported in $5.6 \%-47.0 \%$ of patients $[6,9,10]$. Predisposing factors for thrombosis suggested in the literature $[3,6,11$ 14] include a small diameter of SV and shunt, grade of esophageal varices at presentation, platelet count, an improper lie of the shunt, pathological abnormalities in SV, and the interval between shunt surgery and evaluation for thrombosis. In the early postoperative period, flow and size of vein, the technique of anastomosis, type of suture material, lie of the shunt, and presence of a pathological splenic venous wall can affect shunt thrombosis. In the late phase, underlying pro-coagulant disorder, post-splenectomy thrombocytosis, and decreased flow due to splenectomy are important predisposing factors for shunt thrombosis.

In our study, the difference in confounding factors affecting shunt thrombosis in patient groups with thrombosed versus patent shunt, namely diagnosis of EHPVO and NCPF, grade of esophageal varices at presentation, the diameter of SV and shunt, pathological SV, the technique of anastomosis, the type of suture material used, platelet count, and the interval between shunt surgery and evaluation for thrombosis were not significant (Table 4). Consequently, in this study, we focused on the lie of the shunt.

Sometimes, in conventional PSRS, SV mobilization can result in a long pancreatic tail that hangs and hinders proper anastomosis [15]. The tail of the pancreas is retracted upward at the time of the creation of the shunt. After the shunt has been constructed and the pancreas returns to its normal position, the weight of the distal end of the pancreas may occasionally impinge on the anastomosis, alter its lie, and obliterate the shunt. Based on this observation, we selectively resected the distal-most portion of the pancreatic tail during PSRS, especially in patients having a long pancreatic tail with the tip of

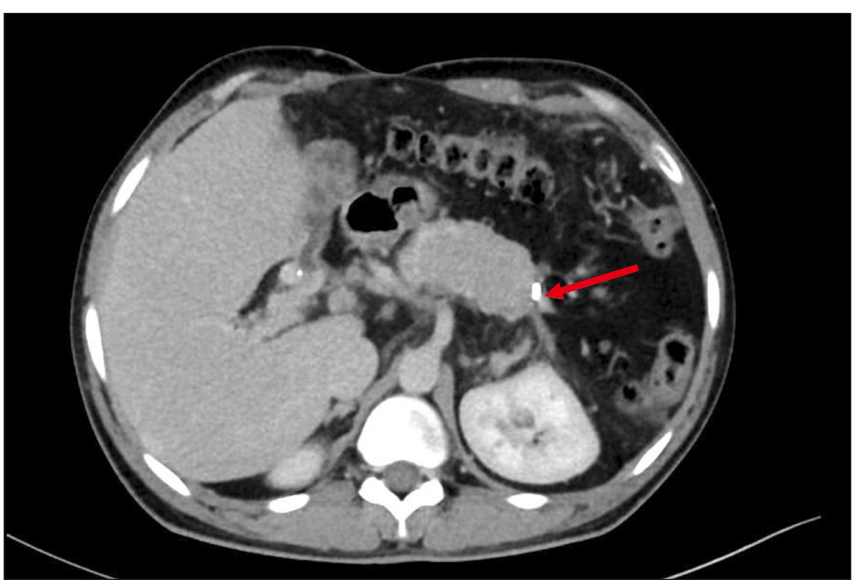

Fig. 6. Postoperative computed tomography portovenogram showing post-caudal pancreatectomy status. Red arrow indicates the cut edge of the pancreas; staples seen.

the pancreatic tail extending up to the splenic hilum (Fig. 1). Only terminal 2 to $3 \mathrm{~cm}$ of the pancreatic tail is resected (Fig. 2, 6). This justifies the use of the term CP rather than distal pancreatectomy.

The incidence of a satisfactory lie of the shunt was higher in Group A $(p=0.04)$ (Table 2$)$. Patients in the study group who developed shunt thrombosis (Group A: 2/17; Group B: 25/67) did not have a satisfactory lie of shunt whereas all patients with a patent shunt (Group A: 15/17; Group B: 42/67) had a satisfactory lie. The greater fall in portal pressure $(p=0.002)$ and the lower shunt thrombosis rate $(p=0.04)$ that we observed (Table 2,3 ) in patients who underwent CP (Group A) might have been due to a satisfactory lie of the shunt.

Mobilization of the SV for a length of $5 \mathrm{~cm}$ is usually done for all PSRS. This is enabled by serially ligating the small collaterals from SV to the pancreas. CP could facilitate this step and prevent occasional large blood losses observed in some patients who underwent conventional PSRS (Table 2). CP using a laparoscopic linear cutter is a relatively easy procedure because the vein and the pancreas are clamped and the pancreas is divided so that the bleeding is minimal. A comparison of the median and range of intraoperative blood loss between the two groups (Table 2) substantiated this assertion. Occasional large blood losses we experienced in Group B were avoided in Group A by CP.

Our results on postoperative pancreatic leak showed that the addition of CP to PSRS did not increase the morbidity of the procedure (Table 3 ).

Unshuntability of the SV has been proposed as a major reason for abandoning shunt during operation. One major reason is that injuries can inflict on the SV during its mobilization from the pancreas. In the presence of a diseased vein wall that occurs in all patients with $\mathrm{NCPH}$ [5], the atheromatous plaque area is a potential focus of tear in the vein. In many situations, 
SV is seen densely adherent to the pancreas. Hence, dissecting the vein out of the pancreas can result in small tears in the vein. The surgeon needs to put all efforts not to injure the pancreas to avoid pancreatic leaks. In patients with densely adherent SV to pancreas, it is easier to excise and remove the pancreas rather than resorting to meticulous dissection of both the SV and the pancreas thus risking tear in the vein. In our experience, addition of $\mathrm{CP}$ significantly contributed to the negligible unshuntability (with CP: 0/17; without CP: 2/69).

Limitations of the present study include the retrospective study design with inherent selection bias and a small number of patients who underwent CP. Of factors influencing shunt thrombosis, a workup for procoagulant disorders was not done. Notwithstanding these limitations, this is the first study to evaluate the role of $\mathrm{CP}$ in shunt patency in patients undergoing PSRS.

In conclusion, $\mathrm{CP}$ is a safe and useful technique for the reduction of the incidence of shunt thrombosis after PSRS in patients with NCPH, especially in those with a long pancreatic tail. This reduction is a consequence of achieving a satisfactory lie of the shunt.

\section{ACKNOWLEDGEMENTS}

The authors thank Dr. Bharath Konan of the Department of Surgical Gastroenterology, Jawaharlal Institute of Postgraduate Medical Education and Research (JIPMER), Puducherry for his support.

\section{FUNDING}

None.

\section{CONFLICT OF INTEREST}

No potential conflict of interest relevant to this article was reported.

\section{ORCID}

Shahana Gupta, https://orcid.org/0000-0003-2876-1701

Biju Pottakkat, https://orcid.org/0000-0002-8474-0270

Raja Kalayarasan, https://orcid.org/0000-0003-4056-8672

Gnanasekaran Senthil, https://orcid.org/0000-0002-8639-5423

Pagadala Naga Balaji Nitesh,

https://orcid.org/0000-0002-9522-7149

\section{AUTHOR CONTRIBUTIONS}

Conceptualization: BP. Data curation: SG, PNBN. Methodology: SG, BP, RK, GS. Visualization: SG, BP, RK. Writing original draft: SG, BP, RK. Writing - review \& editing: BP, RK.

\section{REFERENCES}

1. Pal S, Sahni P. Extrahepatic portal venous obstruction: is the knife irrelevant? Indian J Gastroenterol 2009;28:198-200.

2. Prasad AS, Gupta S, Kohli V, Pande GK, Sahni P, Nundy S. Proximal splenorenal shunts for extrahepatic portal venous obstruction in children. Ann Surg 1994;219:193-196.

3. Mishra PK, Patil NS, Saluja S, Narang P, Solanki N, Varshney V. High patency of proximal splenorenal shunt: a myth or reality? - A prospective cohort study. Int J Surg 2016;27:82-87.

4. Gupta S, Venkata Srinivas G, Chandrasekar AS, Kalayarasan R, Pottakkat B. Splenoadrenal shunt for noncirrhotic portal hypertension. Indian J Surg 2019;81:28-31.

5. Gupta S, Pottakkat B, Verma SK, Kalayarasan R, Chandrasekar AS, Pillai AA. Pathological abnormalities in splenic vasculature in non-cirrhotic portal hypertension: its relevance in the management of portal hypertension. World J Gastrointest Surg 2020;12:1-8.

6. George J, Panwar R, Saluja SS, Sahni P. Surgery for portal hypertension. In: Mishra PK, ed. Textbook of surgical gastroenterology. Vol 2. New Delhi: Jaypee Brothers Medical Publishers, 2016:1357-1366.

7. Bassi C, Marchegiani G, Dervenis C, Sarr M, Abu Hilal M, Adham M, et al. The 2016 update of the International Study Group (ISGPS) definition and grading of postoperative pancreatic fistula: 11 years after. Surgery 2017;161:584-591.

8. Dindo D, Demartines N, Clavien PA. Classification of surgical complications: a new proposal with evaluation in a cohort of 6336 patients and results of a survey. Ann Surg 2004;240:205-213.

9. Dutta HK, Baruah M. Management of extrahepatic portal vein obstruction in children: experience in a tertiary care center in Northeast India. J Med Soc 2015;29:101-105.

10. Bismuth H, Franco D, Alagille D. Portal diversion for portal hypertension in children. The first ninety patients. Ann Surg 1980;192:1824.

11. Orloff MJ, Orloff MS, Girard B, Orloff SL. Bleeding esophagogastric varices from extrahepatic portal hypertension: 40 years' experience with portal-systemic shunt. J Am Coll Surg 2002;194:717-728.

12. Rao KL, Goyal A, Menon P, Thapa BR, Narasimhan KL, Chowdhary SK, et al. Extrahepatic portal hypertension in children: observations on three surgical procedures. Pediatr Surg Int 2004;20:679-684.

13. Wani AH, Shah OJ, Zargar SA. Management of variceal hemorrhage in children with extrahepatic portal venous obstruction-shunt surgery versus endoscopic sclerotherapy. Indian J Surg 2011;73:409-413.

14. Sharma N, Bajpai M, Kumar A, Paul S, Jana M. Portal hypertension: a critical appraisal of shunt procedures with emphasis on distal splenorenal shunt in children. J Indian Assoc Pediatr Surg 2014;19:80-84.

15. Shah OJ, Robbani I. A simplified technique of performing splenorenal shunt (Omar's technique). Tex Heart Inst J 2005;32:549-554. 